Case Report

\title{
Nonarteritic Anterior Ischemic Optic Neuropathy following COVID-19 Vaccination: Consequence or Coincidence
}

\author{
Rika Tsukii, Yuka Kasuya, and Shinji Makino \\ Department of Ophthalmology, Jichi Medical University, Shimotsuke, Tochigi, Japan \\ Correspondence should be addressed to Shinji Makino; makichan@jichi.ac.jp
}

Received 6 September 2021; Revised 27 September 2021; Accepted 5 October 2021; Published 14 October 2021

Academic Editor: Kamal Kishore

Copyright (c) 2021 Rika Tsukii et al. This is an open access article distributed under the Creative Commons Attribution License, which permits unrestricted use, distribution, and reproduction in any medium, provided the original work is properly cited.

\begin{abstract}
To report a patient with nonarteritic anterior ischemic optic neuropathy (NA-AION) occurring soon after the COVID-19 vaccination. A 55-year-old woman presented with a 4-day history of inferior visual field disturbance in the right eye 7 days after receiving the first dose of Pfizer-BioNTech COVID-19 vaccine. Examination revealed a best-corrected visual acuity of $20 / 20$ in both eyes. A relative afferent pupillary defect was observed in the right eye. Fundoscopy revealed diffuse optic disc swelling in the right eye, which was prominent above the optic disc. Goldmann visual field testing identified an inferior altitudinal visual field defect with I/2 isopter in the right eye. Although typical complete inferior visual field defect was not detected, a diagnosis of NA-AION was made. The patient was followed without any treatment. During the 2-month follow-up period, the optic disc swelling was gradually improved, and visual acuity was maintained 20/20; however, the optic disc looked diffusely pale in the right eye. Although it is uncertain whether the development of NA-AION after COVID-19 vaccination was consequential or coincidental, we speculate that the close temporal relationship with COVID-19 vaccination suggests the possibility of vasculopathy on the microvascular network of optic nerve head as background of inflammatory or immunemediated element to the timing of the onset of NA-AION. The aim of this case report is to present this biological plausibility and to elucidate potential ophthalmological complications.
\end{abstract}

\section{Introduction}

Severe acute respiratory syndrome coronavirus 2 (SARSCoV-2) infection and the resulting coronavirus disease 2019 (COVID-19) pandemic hit the world by storm, and Japan was no exception. Currently, there has been a tremendous increase in reports of ophthalmic manifestations related with COVID-19 and its vaccination [1-3].

Nonarteritic anterior ischemic optic neuropathy (NAAION) is an important cause of acute visual loss in middle-aged and elderly populations [4]. Typically, NAAION is associated with risk factors such as systemic hypertension, diabetes mellitus, and optic disc morphology (small and crowded optic disc) [4].

Here, we report a case of NA-AION occurring soon after the COVID-19 vaccination.

\section{Case Presentation}

A 55-year-old woman presented with a 4-day history of inferior visual field disturbance in the right eye 7 days after receiving the first dose of Pfizer-BioNTech COVID-19 vaccine (BNT162b2 mRNA COVID-19 vaccine). Her personal and family histories as well as physical examination results were unremarkable. She had no other health complaints. Examination revealed a best-corrected visual acuity of $20 / 20$ in both eyes. A relative afferent pupillary defect was observed in the right eye. There was no abnormal ocular motility in either eye. Fundoscopy revealed diffuse optic disc swelling in the right eye, which was prominent above the optic disc (Figure 1(a)). In contrast, no abnormal findings were observed in the left eye (Figure 1(b)). Optical coherence tomography confirmed optic disc swelling in the right eye. 


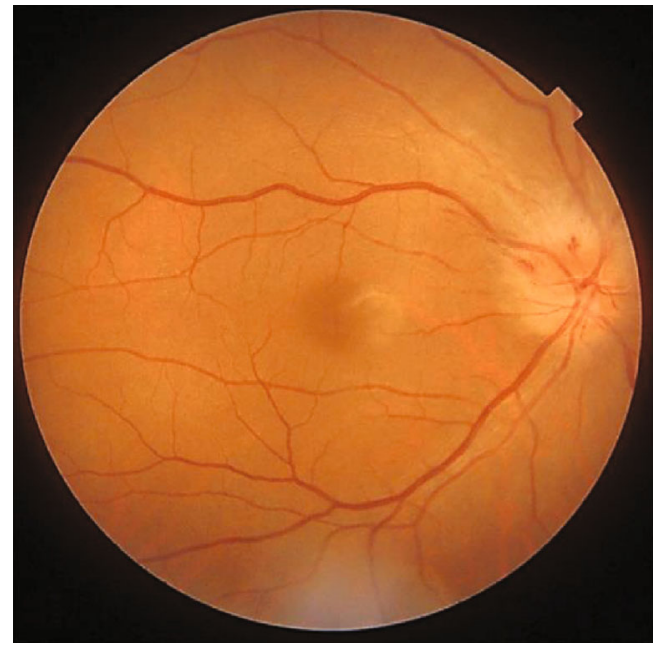

(a)

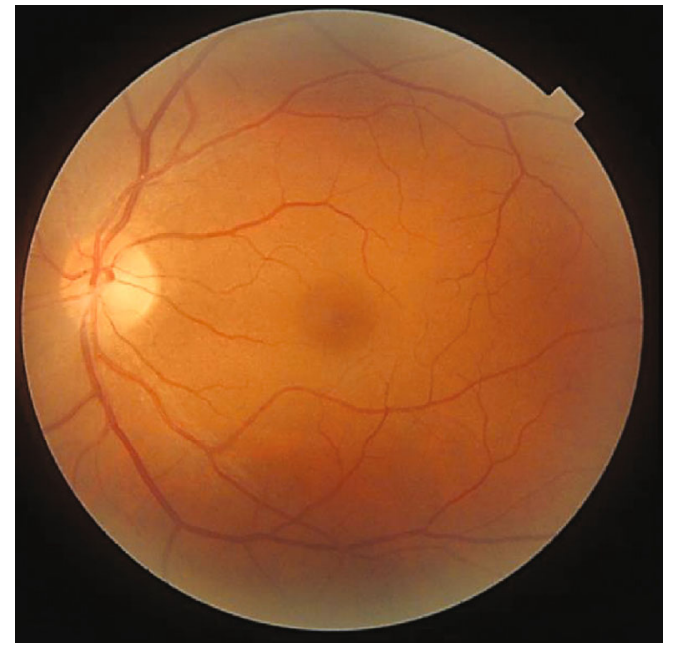

(b)

Figure 1: Photographs of the right (a) and left (b) fundus on initial examination. Note the diffuse optic disc swelling in the right eye, which was prominent above the optic disc.

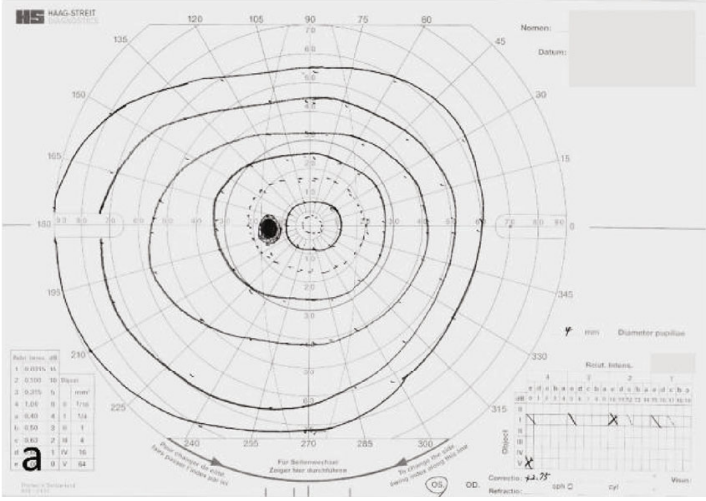

(a)

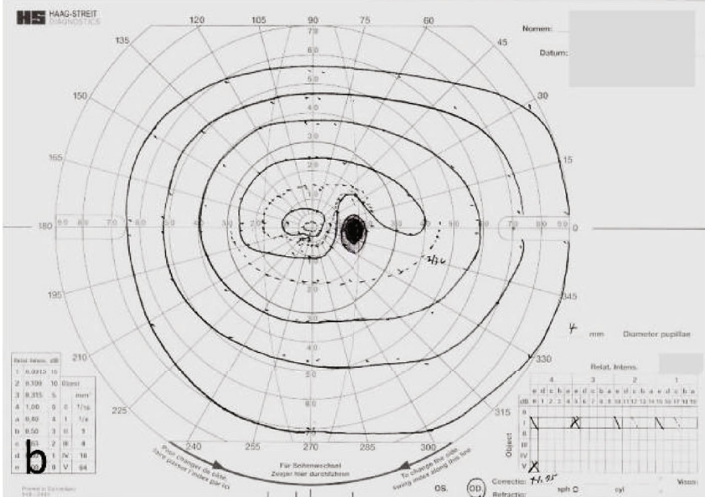

(b)

FIGURE 2: Goldmann perimetry of the left (a) and right (b) eyes. Note the inferior altitudinal visual field defect with I/2 isopter in the right eye.

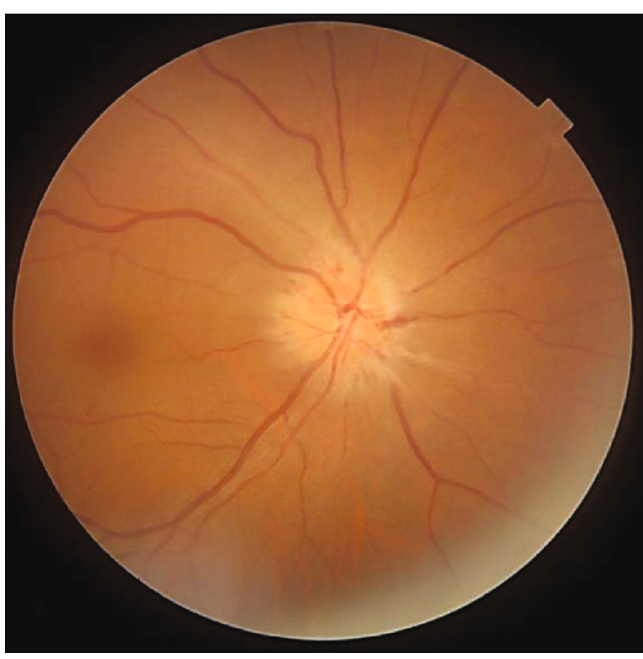

(a)

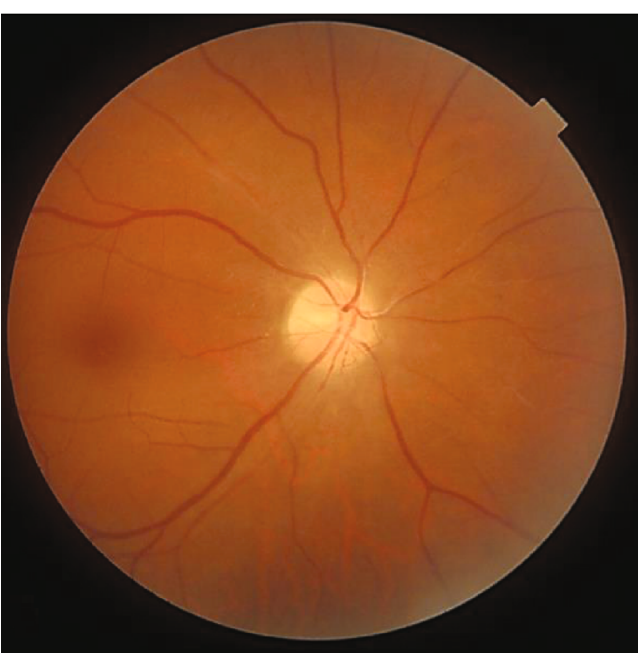

(b)

FIGURE 3: Photographs of the right fundus on 2 weeks (a) and 2 months (b) after the initial examination. Note the optic disc swelling was gradually improved; however, the optic disc looked diffusely pale. 
However, no abnormal findings were observed in the right macula. Goldmann visual field testing identified an inferior altitudinal visual field defect with I/2 isopter in the right eye (Figure 2). The average critical flicker frequency value was $34 \mathrm{~Hz}$ in the right eye and $37 \mathrm{~Hz}$ in the left eye. Cranial and orbital MRI showed no abnormal findings. Laboratory examination revealed erythrocyte sedimentation rate was $11 \mathrm{~mm} / \mathrm{h}$, and C-reactive protein (CRP) level was $0.02 \mathrm{mg} / \mathrm{L}$ (reference range; 0-0.14). Myeloperoxidaseantineutrophil cytoplasmic antibody (MPO-ANCA) was $16.0 \mathrm{U} / \mathrm{mL}$ (reference range, $<3.5$ ) and proteinase 3- (PR3-) ANCA was $1.0 \mathrm{U} / \mathrm{mL}$ (reference range, <3.5). Fluorescein angiography, color vison test, cardiac echography, carotid doppler, and specialized blood coagulation examination were not available in this case.

Although typical complete inferior visual field defect was not detected nor above-mentioned examinations were not conducted, based on patient's history and the aforementioned examinations, a diagnosis of NA-AION was made. The patient was followed without any treatment. During the 2-month follow-up period, the optic disc swelling was gradually improved, and visual acuity was maintained 20/20; however, the optic disc looked diffusely pale in the right eye (Figure 3).

\section{Discussion}

NA-AION is thought to develop due to circulatory insufficiency of the posterior ciliary arteries supplying the optic nerve. Having small, crowded optic disc or other vasculopathies are risk factors for developing NA-AION. Although in the present case, the relatively young patient did not have any vasculopathic risk factors, she developed NA-AION following COVID-19 vaccination.

The occurrence and significance of autoimmune manifestations after the administration of viral vaccines remain controversial. Only two previous cases of NA-AION following influenza vaccination have been reported in the literature $[5,6]$. These authors proposed an immune complexmediated vasculopathy as a likely mechanism.

Previous cases of NA-AION in the setting of COVID-19 infection were reported in the literature [7-9]. SARS-CoV-2 can cause significant inflammation resulting in hypercoagulability manifesting as pulmonary embolism, deep-vein thrombosis, ischemic strokes, or myocardial infarcts [7]. As mentioned, patients with COVID-19 infection can manifest with hypercoagulability and hypoxemia, both of which may contribute to the development of NA-AION.

In contrast, various adverse effects have been reported with COVID-19 vaccine [2, 3], but these events' specific mechanism and frequency have not been investigated thoroughly. A previous case of arteritic anterior ischemic optic neuropathy following COVID-19 vaccination was reported [10]; however, this is the first reported case of NA-AION which was temporally related to the COVID-19 vaccination in Japan.

COVID-19 vaccines result in producing high levels of neutralizing antibodies after injection. These neutralizing antibodies recognize and target the spike proteins in the virus, killing it before the virus is disseminated and cause illness [10]. Neutralizing antibodies against SARS-CoV-2 spike proteins and/or activated T-helper-1 cells after vaccination can crossreact with proteins and antigens in large arteries, outer retinal layers, and retinal pigment epithelial cell [10]. Although it is uncertain whether the development of NA-AION after COVID-19 vaccination was consequential or coincidental, we speculate that the close temporal relationship with COVID-19 vaccination suggests the possibility of vasculopathy on the microvascular network of optic nerve head as background of inflammatory or immunemediated element to the timing of the onset of NA-AION.

Finally, the aim of this case report is to present this biological plausibility and to elucidate potential ophthalmological complications.

\section{Ethical Approval}

The identity of the patient has been kept anonymous in this case report.

\section{Consent}

We obtained written and informed consent from the patient for the publication of case details and images.

\section{Conflicts of Interest}

The authors have no financial interests to disclose.

\section{References}

[1] M. Sen, S. G. Honavar, N. Sharma, and M. S. Sachdev, "COVID-19 and eye: a review of ophthalmic manifestations of COVID-19," Indian Journal of Ophthalmology, vol. 69, no. 3, pp. 488-509, 2021.

[2] N. G. Kounis, I. Koniari, C. de Gregorio et al., "Allergic reactions to current available COVID-19 vaccinations: pathophysiology, causality, and therapeutic considerations," Vaccines, vol. 9, no. 3, p. 221, 2021.

[3] C. Y. C. Chau, L. L. W. Chow, S. Sridhar, and K. C. Shih, "Ophthalmological considerations for COVID-19 vaccination in patients with inflammatory eye diseases and autoimmune disorders," Ophthalmology and Therapy, vol. 10, no. 2, pp. 201209, 2021.

[4] S. S. Hayreh, "Ischemic optic neuropathy," Progress in Retinal and Eye Research, vol. 28, no. 1, pp. 34-62, 2009.

[5] A. Kawasaki, V. A. Purvin, and R. Tang, "Bilateral anterior ischemic optic neuropathy following influenza vaccination," Journal of Neuro-Ophthalmology, vol. 18, no. 1, pp. 56-59, 1998.

[6] G. Manasseh, D. Donovan, E. H. Shao, and S. R. Taylor, "Bilateral sequential non-arteritic anterior ischaemic optic neuropathy following repeat influenza vaccination," Case reports in Ophthalmology, vol. 5, no. 2, pp. 267-269, 2014.

[7] J. Rho, S. C. Dryden, C. D. McGuffey, B. T. Fowler, and J. Fleming, "A case of non-arteritic anterior ischemic optic neuropathy with COVID-19," Cureus, vol. 12, article e11950, 2020.

[8] L. Moschetta, G. Fasolino, and R. W. Kuijpers, "Non-arteritic anterior ischaemic optic neuropathy sequential to SARS- 
CoV-2 virus pneumonia: preventable by endothelial protection?," BML Case Reports, vol. 14, no. 7, article e240542, 2021.

[9] K. M. Clarke, V. Riga, A. L. Shirodkar, and J. Meyer, "Proning related bilateral anterior ischaemic optic neuropathy in a patient with COVID-19 related acute respiratory distress syndrome," BMC Ophthalmology, vol. 21, no. 1, p. 276, 2021.

[10] A. Maleki, S. Look-Why, A. Manhapra, and C. S. Foster, "COVID-19 recombinant mRNA vaccines and serious ocular inflammatory side effects: real or coincidence?," Journal of Ophthalmic \& Vision Research, vol. 16, no. 3, pp. 490-501, 2021. 